\title{
Derivation and Validation of a Predictive Score for Disease Worsening in Patients with COVID-19
}

\author{
Grigoris T. Gerotziafas ${ }^{1,2(0)}$ Theodoros N. Sergentanis $^{3}$ Guillaume Voiriot ${ }^{4}$ Ludovic Lassel $^{5}$ \\ Chryssa Papageorgiou ${ }^{6}$ Alexandre Elabbadi ${ }^{4}$ Matthieu Turpin ${ }^{4}$ Patrick Vandreden ${ }^{7}$ \\ Loula Papageorgiou ${ }^{1,2}$ Theodora Psaltopoulou ${ }^{3}$ Evangelos Terpos ${ }^{3}$ \\ Meletios-Athanasios Dimopoulos ${ }^{3}$ Antoine Parrot $^{8}$ Jacques Cadranel ${ }^{8}$ Gilles Pialoux ${ }^{5}$ \\ Muriel Fartoukh ${ }^{4}$ Ismail Elalamy ${ }^{1,2}$
}

\footnotetext{
${ }^{1}$ Research Group "Cancer, Haemostasis and Angiogenesis," INSERM U938, Centre de Recherche Saint-Antoine, Institut Universitaire de Cancérologie, Faculty of Medicine, Sorbonne University, Paris, France

2 Service d'Hématologie Biologique Hôpital Tenon, Hôpitaux Universitaires de l'Est Parisien, Assistance Publique Hôpitaux de Paris, Faculté de Médecine Sorbonne Université, Paris, France

${ }^{3}$ Department of Clinical Therapeutics, School of Medicine, National and Kapodistrian University of Athens, Athens, Greece

${ }^{4}$ Sorbonne Université, Service de Médecine Intensive Réanimation, Hôpital Tenon, Assistance Publique - Hôpitaux de Paris, Paris, France ${ }^{5}$ Service des Maladies Infectieuses et Tropicales, Hôpital Tenon, Hôpitaux Universitaires de l'Est Parisien, Assistance Publique Hôpitaux de Paris, Faculté de Médecine Sorbonne Université, Paris, France

${ }^{6}$ Service Anesthésie, Réanimation et Médecine Périopératoire, Hôpital Tenon, Hôpitaux Universitaires de l'Est Parisien, Assistance Publique Hôpitaux de Paris, Faculté de Médecine Sorbonne Université, Paris, France

${ }^{7}$ Clinical Research Department, Diagnostica Stago, Gennevilliers, France

${ }^{8}$ Service de pneumologie, Hôpital Tenon, Hôpitaux Universitaires de l'Est Parisien, Assistance Publique Hôpitaux de Paris, Faculté de Médecine Sorbonne Université, Paris, France
}

Address for correspondence Grigoris T. Gerotziafas, MD, PhD, INSERM U938 Bâtiment Kourilsky, 34 rue Crozatier, Hôpital Saint Antoine, Paris, Cedex 75012, France (e-mail: grigorios.gerotziafas@inserm.fr).
Abstract
Keywords
- COVID-19
- SARS-CoV-2
- disseminated intravascular coagulation
- antithrombin
- D-dimers

The prospective observational cohort study COMPASS-COVID-19 aimed to develop a risk assessment model for early identification of hospitalized COVID-19 patients at risk for worsening disease. Patients with confirmed COVID-19 $(n=430)$ hospitalized between March 18 and April 21, 2020 were divided in derivation $(n=310)$ and validation ( $n=120)$ cohorts. Two groups became evident: (1) good prognosis group (G-group) with patients hospitalized at the conventional COVID-19 ward and (2) Worsening disease group (W-group) with patients admitted to the intensive care unit (ICU) from the emergency departments. The study end point was disease worsening (acute respiratory failure, shock, myocardial dysfunction, bacterial or viral coinfections, and acute kidney injury) requiring ICU admission. All patients were routinely evaluated for full blood count, prothrombin time, fibrinogen, D-dimers, antithrombin (AT), and protein $C$ activity. Data from the first hospitalization day at the conventional ward or the ICU were analyzed. Cardiovascular risk factors and comorbidities were routinely registered. Obesity, hypertension, diabetes and male gender, increased fibrinogen and received

May 18,2020

accepted after revision

August 13, 2020 (c) 2020. Thieme. All rights reserved. Georg Thieme Verlag KG,

Rüdigerstraße 14,

70469 Stuttgart, Germany
DOI https://doi.org/

10.1055/s-0040-1716544. ISSN 0340-6245. 
D-dimers, thrombocytopenia, AT deficiency, lymphopenia, and an International Society on Thrombosis and Haemostasis (ISTH) score for compensated disseminated intravascular coagulation score (CDIC-ISTH) $\geq 5$ were significant risk factors for worsening disease. The COMPASS-COVID-19 score was derived from multivariate analyses and includes obesity, gender, hemoglobin, lymphocyte, and the CDIC-ISTH score (including platelet count, prothrombin time, D-dimers, AT, and protein C levels). The score has a very good discriminating capacity to stratify patients at high and low risk for worsening disease, with an area under the receiver operating characteristic curve value of 0.77 , a sensitivity of $81 \%$, and a specificity of $60 \%$. Application of the COMPASS-COVID-19 score at the validation cohort showed $96 \%$ sensitivity. The COMPASS-COVID-19 score is an accurate clinical decision-making tool for an easy identification of COVID-19 patients being at high risk for disease worsening.

\section{Introduction}

Severe acute respiratory syndrome coronavirus 2 (SARSCoV-2) causes coronavirus disease 2019 (COVID-19). ${ }^{1-3}$ The SARS-CoV-2 pandemic has put the health care systems worldwide under extremely high stress. COVID-19 is characterized by acute pneumonia which may progress to respiratory failure and life-threatening complications, including acute respiratory distress syndrome and multisystem organ failure with fatal outcome. ${ }^{4,5}$ COVID-19 should be regarded as a systemic disease involving multiple systems. ${ }^{6-8}$ COVID19 is associated with excessive inflammation, platelet activation, endothelial dysfunction, blood coagulation activation, and fibrin formation. ${ }^{9-11}$ Current management of patients hospitalized in an intensive care unit (ICU) is based on supportive care and the mortality rate may be high. ${ }^{12,13}$ Blood hypercoagulability-documented by an increase in Ddimers and a decrease in antithrombin (AT)-is frequently encountered among COVID-19 patients. ${ }^{14}$

Identification of patients with COVID-19 being at high risk for clinical deterioration is a challenging issue for an earlier adapted treatment and positive clinical outcome. ${ }^{15,16}$ These patients could benefit from earlier appropriate oxygen support, antithrombotic agents, and compassionate-use therapies, including antiretrovirals, anti-inflammatory drugs, immunomodulatory compounds, and convalescent plasma. ${ }^{17-21}$

In this prospective observational study conducted at the COVID-19 center of Tenon University Hospital in Paris, we aimed to identify the most relevant clinical and hematological risk factors for worsening of COVID-19 by constructing an accurate risk assessment tool.

\section{Methods}

\section{Participants}

In the set-up of the COVID-19 center at Tenon University Hospital (APHP.6, Sorbonne University, Paris), we designed a prospective observational cohort study and enrolled all admitted patients in two phases: (1) between March 18 and April 5, 2020 to constitute the derivation cohort and (b) between April 6 and April 21 to constitute the validation cohort which was composed only of new patients. According to the follow-up during the course of the disease, two groups became evident: (1) good prognosis group (G-group) with patients hospitalized at the conventional COVID-19 ward. G-group patients were assessed on the first admission day. (2) Worsening disease group (W-group) included patients admitted to the ICU from the emergency departments since they presented with clinically deteriorated COVID-19. W-group patients were assessed on the first day of admission.

\section{Definitions}

All patients had laboratory-confirmed COVID-19 infection and were hospitalized either in the conventional COVID-19 ward of the medical department or in the COVID-19 ICU. A confirmed case of COVID-19 was defined by a positive result on a reverse transcriptase polymerase chain reaction (rtPCR) assay from a specimen collected on a nasopharyngeal swab and imaging of well-documented pneumonia according the Fleischner Society consensus statement. ${ }^{22}$ Pregnant women, patients receiving anticoagulant treatment, and patients with cytopenia due to current anticancer treatment were excluded. All patients hospitalized in the conventional medical department or in the ICU routinely received thromboprophylaxis with body-weight-adapted enoxaparin. All clinical and biological data were crosschecked with the electronic files using the ORBIS software (Agfa Healthcare) and the GLIMS laboratory information system (MIPS France) of Tenon University Hospital.

\section{Hematological Parameters}

In the set-up of the COVID-19 center at Tenon University Hospital, all patients were evaluated daily with a "COAGCOVID" panel composed of tests of various parameters: prothrombin time (PT), fibrinogen, D-dimers, AT activity, protein $\mathrm{C}(\mathrm{PC})$ activity, and platelet count. These tests are predictors of compensated disseminated intravascular coagulation (DIC) according to the International Society on Thrombosis and Haemostasis (ISTH) score (compensated DIC-ISTH). ${ }^{23}$ In addition to platelets, all other hemogram 
parameters were also analyzed. Blood samples were routinely obtained via atraumatic antecubital venipuncture or from the central vein catheter. For coagulation tests, blood was collected in $3.5 \mathrm{~mL}$ Vacuette tubes containing $0.109 \mathrm{~mol} / \mathrm{L}$ trisodium citrate-one volume trisodium citrate to nine volumes of blood-(Greiner Bio-One, Courtaboeuf, France) and centrifuged at $2,000 \mathrm{~g}$ for 20 minutes at room temperature for platelet-poor plasma (PPP) preparation. Within 30 minutes upon preparation, PPP samples were assessed for blood coagulation tests on the STA-R Max instrument from Stago (Asnières-sur-Seine, France) according to manufacturer's instructions. PT was assessed with chronometric assay using the STA-NeoPTimal reagent (ref: 01165). Fibrinogen was measured with the Clauss-based chronometric assay using the Liquid FIB reagent (ref: 00673). D-dimers were measured with turbidimetric assay using the STALiatest D-Di Plus reagent (ref: 00662). AT and PC activities were measured with the amidolytic assays STACHROM ATIII (ref: 00596, from Stago, Asnières, France) and BIOPHEN PC (LRT) (ref. 221205, from Hyphen BioMed, Neuville-sur-Oise, France), respectively. Hemogram parameters and platelet count were assessed on whole blood collected in $4 \mathrm{~mL}$ EDTA BD Vacutainer tubes (Becton-Dickinson, Le Pont-deClaix, France) using the Sysmex XN-3100 instrument (Paris, France). All hematological tests were performed at the ISO certified Central Haematological Laboratory of Thrombosis Center at Tenon University Hospital in Paris.

\section{Ethics}

The protocol of the study was in accordance with the commitment of the Helsinki Declaration and all patients received care according to the recommended institutional practice during the COVID-19 pandemic. All hematological tests were performed in the frame of routine monitoring of patients as decided by the local institutional board for the management of the COVID-19 patients. This study was approved by the institutional ethics board. The observational design of the study did not impose the need for getting informed consent from individual patients.

\section{Outcomes}

The study end point was disease worsening requiring ICU admission. Disease worsening requiring ICU admission was defined according to the following criteria ${ }^{16}$ : acute hypoxemic respiratory failure judged on the basis of increased need for oxygen supply more than $9 \mathrm{~L} /$ minute or clinical signs of respiratory insufficiency (shortness of breath, respiratory rate $\geq 30$ times $/ \mathrm{min}$ ), arterial oxygen saturation (resting status) $\leq 92 \%$, shock, myocardial dysfunction, bacterial or viral coinfections, and acute kidney injury.

\section{Statistical Analysis}

Data from the first hospitalization day for the G-group and the first day on ICU admission for the W-group were analyzed. The normal ranges of the COAG-COVID panel of tests have been established by the Thrombosis Center of Tenon University Hospital, according to the requirements for the good quality of laboratory practice. The number of patients included in the derivation cohort was calculated according to the following assumptions: (1) the model had to be constructed according to the rule of thumb, the so-called events per variable (EPV) $10-1$ and (2) less than 10 variables should be included in the model in order for it to be easy to use. ${ }^{24-26}$ Thus, at least 70 patients were required to be enrolled in the $\mathrm{W}$-group to respond to the above conditions accommodating at maximum a seven-variable model. Continuous variables were summarized as median (interquartile range) and categorical variables as frequency and percentage. Because of the deviation from normality (as evidenced by the Shapiro-Wilk test), the comparison of continuous variables between patients with worsening disease and those hospitalized at the conventional ward was performed using the MannWhitney-Wilcoxon test for independent samples. Regarding the associations between disease worsening and hematological parameters at baseline, the latter were converted to binary variables on the basis of laboratory normal values. Univariate and multivariate logistic regression analyses were performed to evaluate the independent associations between disease worsening (dependent variable) and the examined hematological parameters (independent variables) as binary variables: 1 (yes) or 0 (no). In the multivariate approach aiming to create a score predicting disease worsening, stepwise selection of variables was performed on the basis of the Akaike information criterion (AIC). To obtain the score, $\beta$ coefficients of the final logistic regression model were rounded and rescaled from the logarithm of the odds ratios (ORs). Receiver operating characteristic (ROC) curve analysis was subsequently undertaken; the area under the ROC curve (AUC) was estimated to evaluate model discrimination performance. The optimal cut-off level was identified through the maximization of unweighted Youden's index by calculating sensitivity, specificity, positive predictive value, (PPV) and negative predictive value (NPV). Calibration of the model was examined with the Hosmer-Lemeshow test and the respective plot of expected versus observed probability was constructed, for the G-group and W-group. The level of statistical significance was set at 0.05 . Data were analyzed using the STATA/SE version 13 statistical software (Stata Corp., College Station, Texas, United States).

\section{Results}

\section{Derivation Cohort}

Among 330 patients with confirmed COVID-19 disease, 310 patients who responded to the inclusion criteria were enrolled in the derivation cohort. The remaining 20 patients were excluded because of pregnancy $(n=5)$, pancytopenia due to chemotherapy ( $n=6)$, or oral anticoagulant treatment with direct oral anticoagulants or vitamin $\mathrm{K}$ antagonists $(n=9)$. The G-group included 208 patients. The W-group consisted of 102 patients; $87 \%$ of these patients were admitted to the ICU directly from the emergency department. Males were 113 out of 208 patients in the G-group and 76 out of 102 patients in the W-group. Age ranged from 19 to 95 years in the G-group and from 30 to 93 years in the W-group. 


\section{Validation Cohort}

The validation cohort included 120 patients stratified in the G-group $(n=89)$ and the $\mathrm{W}$-group $(n=31) ; 90 \%$ of patients in the W-group were admitted to the ICU directly from the emergency department. Males were 58 out of 89 patients in the G-group and 25 out of 31 patients in the W-group. Age ranged from 21 to 95 years in the G-group and from 30 to 98 years in the $\mathrm{W}$-group.

The derivation and validation cohorts were comparable regarding the age and sex distribution in each one of the two groups (G- and W-groups). Detailed epidemiological, clinical, and hematological characteristics of the derivation and validation cohorts are shown in -Tables $\mathbf{1}$ and $\mathbf{2}$. Very few patients received compassionated or antiviral treatments. Among the G-group patients, five received treatment with lopinavir-ritonavir, one was treated with remdesivir, and another one received hydroxychloroquine. Among the patients in W-group, only four received lopinavir-ritonavir. Upon hospitalization all patients received thromboprophylaxis with low-molecularweight heparin (LMWH; enoxaparin). The dose of enoxaparin was adapted according to the evolution of D-dimers and the levels of the anti-Xa activity. Moreover, patients with AT deficiency (AT activity lower than 50\%) upon admission or during hospitalization received treatment with AT concentrate according to the protocol published elsewhere. ${ }^{14}$

In both cohorts, there were no missing values of the COAG-COVID biomarkers and clinical predictors. A follow- up of at least 12 days was predicted. However, the follow-up for the most recently enrolled patients was shorter because France was still at the peak of COVID-19 public health crisis when the database closed and data analysis was performed. Male gender representation was significantly higher in the W-group as compared with the G-group. Obesity (body mass index $[\mathrm{BMI}]>30$ ), arterial hypertension, and diabetes were significantly more frequent in the $\mathrm{W}$-group as compared with the G-group. Chronic renal insufficiency and cardiovascular disease were more frequent in the W-group as compared with the G-group. Interestingly, very few patients in both groups were active smokers. Data are summarized in - Table 1.

\section{Derivation of the COMPASS-COVID-19 Risk Assessment Model}

In the derivation cohort, compensated DIC was diagnosed in $8.2 \%$ of patients in the G-group and in $28.2 \%$ of patients in the W-group ( $p=0.001$; - Table 1). Compared with the G-group, the patients in the W-group had significantly lower levels of AT, PC, platelets, lymphocyte, monocyte, and red blood cell counts, as well as hemoglobin and hematocrit. They had significantly higher levels of fibrinogen, D-dimers, white blood cells, and neutrophil counts. No difference was noted in eosinophil and basophil counts. Data are summarized in -Table 2.

Table 1 Demographic data, cardiovascular risk factors, comorbidities, and DIC rates in hospitalized COVID-19 patient enrolled in the derivation cohort hospitalized in conventional ward (G-group) or presenting worsening disease (W-group)

\begin{tabular}{|c|c|c|c|c|c|c|}
\hline & \multicolumn{3}{|l|}{ Derivation cohort } & \multicolumn{3}{|c|}{ Validation cohort } \\
\hline & G-group & W-group & \multirow[t]{2}{*}{$p$-Value } & G-group & W-group & $p$-Value \\
\hline & $(n=208)$ & $(n=102)$ & & $(n=89)$ & $(n=31)$ & \\
\hline \multicolumn{7}{|l|}{ Demographics } \\
\hline Gender male & $54.3 \%(113 / 208)$ & $74.5 \%(76 / 102)$ & - & $66.3 \%(59 / 89)$ & $80.6 \%(25 / 31)$ & \\
\hline Age $(y)^{a}$ & $\begin{array}{l}\text { 19-95 } \\
\text { (median: 66) }\end{array}$ & $\begin{array}{l}30-93 \\
\text { (median: 61) }\end{array}$ & 0.06 & $\begin{array}{l}21-95 \\
\text { (median: 63) }\end{array}$ & $\begin{array}{l}30-80 \\
\text { (median: 59) }\end{array}$ & 0.06 \\
\hline \multicolumn{7}{|l|}{ Cardiovascular risk factors } \\
\hline Hypertension & $39.5 \%(82 / 208)$ & $64.6 \%(66 / 102)$ & $<0.001$ & $42.7 \%(38 / 89)$ & $61.3 \%(19 / 31)$ & 0.001 \\
\hline Diabetes & $15.8(33 / 208)$ & $33.3 \%(34 / 102)$ & 0.006 & 21.3\% (19/89) & $12.9 \%(4 / 31)$ & 0.01 \\
\hline Obesity & $7.8 \%(16 / 208)$ & $31.5 \%(32 / 102)$ & $<0.001$ & $10.1 \%(9 / 89)$ & $32.2 \%(10 / 31)$ & $<0.001$ \\
\hline Active smoking & $4.4 \%(9 / 208)$ & $3.9 \%(4 / 102)$ & 0.612 & $4.5 \%(4 / 89)$ & $3.2 \%(1 / 31)$ & 0.5 \\
\hline \multicolumn{7}{|l|}{ Comorbidities } \\
\hline Chronic renal disease & $7.3 \%(17 / 208)$ & $18.8 \%(19 / 102)$ & 0.003 & $11.2 \%(10 / 89)$ & $19.4 \%(6 / 31)$ & 0.002 \\
\hline $\begin{array}{l}\text { Chronic obstructive } \\
\text { pulmonary disease }\end{array}$ & $8.3 \%(17 / 208)$ & $10.7 \%(11 / 102)$ & 0.548 & $12.4 \%(11 / 89)$ & $15.1 \%(5 / 31)$ & 0.62 \\
\hline Active cancer & $12.6 \%(26 / 208)$ & $1.9 \%(2 / 102)$ & 0.003 & $10,1 \%(9 / 89)$ & $3.2 \%(1 / 31)$ & 0.001 \\
\hline End-stage renal disease & $3.9 \%(8 / 208)$ & $8.8 \%(9 / 102)$ & 0.105 & $2.2 \%(2 / 89)$ & $9.7 \%(3 / 31)$ & 0.051 \\
\hline \multicolumn{7}{|l|}{ Coagulopathy } \\
\hline $\begin{array}{l}\text { Compensated DIC } \\
\text { (DIC-ISTH score } \geq 5 \text { ) }\end{array}$ & $8.2 \%(17 / 208)$ & $28.4 \%(29 / 102)$ & $<0.001$ & $2.2 \%(2 / 89)$ & $3.2 \%(1 / 31)$ & 0.3 \\
\hline
\end{tabular}

Abbreviations: BMI, body mass index; DIC, disseminated intravascular coagulation; ISTH, International Society on Thrombosis and Haemostasis; VTE, venous thromboembolism.

a Values for age are in the minimum and maximum range. 


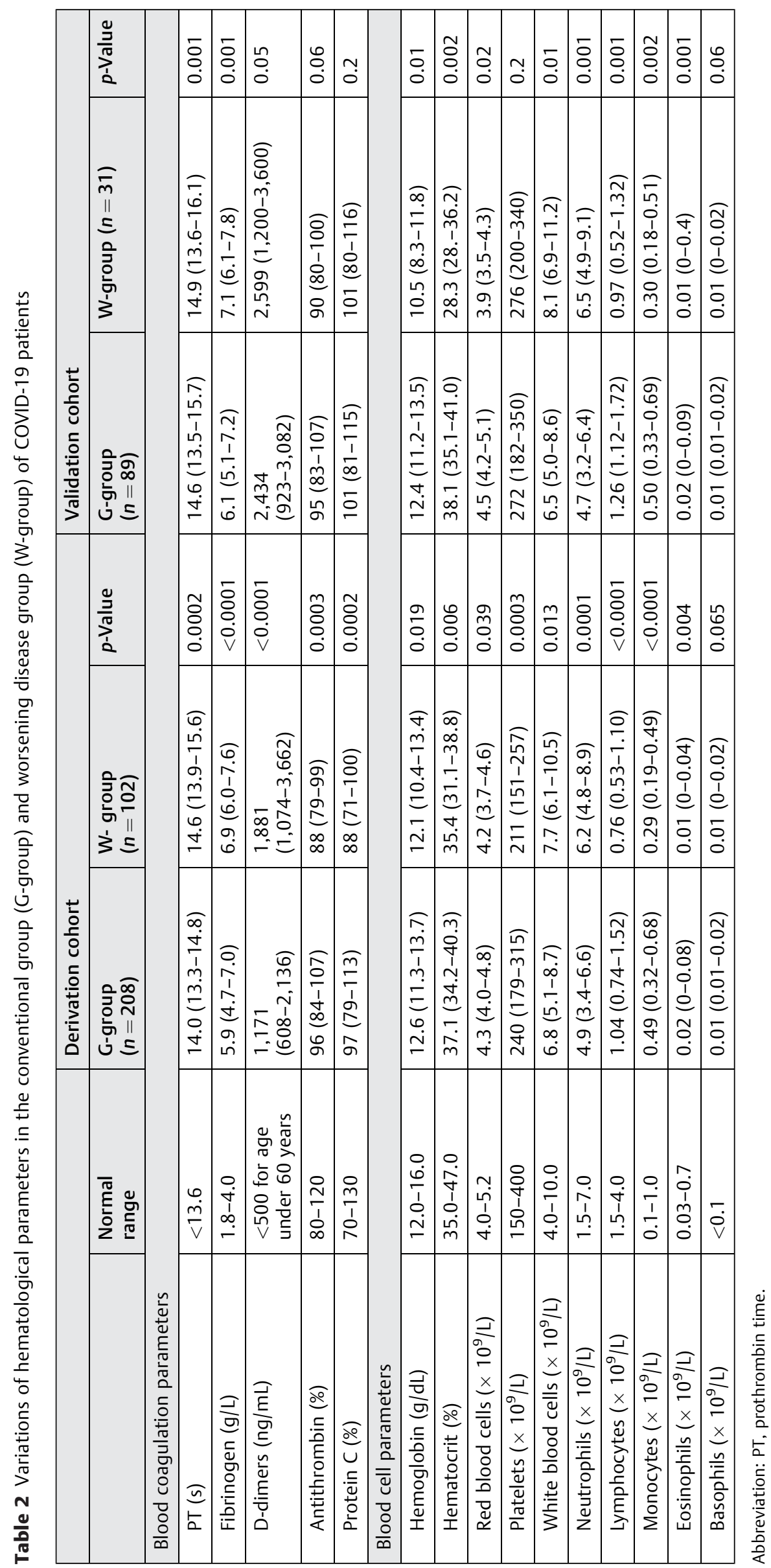




\section{Clinical Predictors for Disease Worsening}

The univariate analysis showed that the risk for worsening disease was increased in men compared with women: $\mathrm{OR}=2.43,95 \%$ confidence interval $(\mathrm{CI}): 1.44$ to 4.10 . Among clinical predictors, obesity ( $\mathrm{OR}=5.44,95 \% \mathrm{CI}$ : 2.77-10.67), hypertension ( $\mathrm{OR}=2.79,95 \% \mathrm{CI}: 1.69-4.62)$, and diabetes
$(\mathrm{OR}=2.13,95 \% \mathrm{CI}: 1.23-3.69)$ were significantly related with an increased risk for disease worsening. Surprisingly current smoking was not a significant risk factor for clinical deterioration of COVID-19 patients. Among comorbidities, chronic kidney disease $(\mathrm{OR}=2.92,95 \% \mathrm{Cl}: 1.40-6.09)$ was a risk factor for disease worsening (-Table 3 ).

Table 3 Univariate analysis of COVID-19 patients determining the risk factors associated with worsening disease

\begin{tabular}{|c|c|c|c|}
\hline Examined parameters & Compared categories & OR $(95 \% \mathrm{Cl})$ & $p$-Value \\
\hline \multicolumn{4}{|l|}{ Demographics } \\
\hline Gender & Male vs. female & $2.43(1.44-4.10)$ & 0.001 \\
\hline Age $(y)$ & $\geq 70$ vs. $<70$ & $0.48(0.28-0.81)$ & 0.006 \\
\hline \multicolumn{4}{|l|}{ Cardiovascular risk factors } \\
\hline Obesity & Yes vs. no & $5.44(2.77-10.67)$ & $<0.001$ \\
\hline Hypertension & Yes vs. no & $2.79(1.69-4.62)$ & $<0.001$ \\
\hline Diabetes mellitus & Yes vs. no & $2.13(1.23-3.69)$ & 0.007 \\
\hline Current smoking & Yes vs. no & $0.71(0.19-2.68)$ & 0.614 \\
\hline \multicolumn{4}{|l|}{ Comorbidities } \\
\hline Chronic kidney disease & Yes vs. no & $2.92(1.40-6.09)$ & 0.004 \\
\hline $\begin{array}{l}\text { Chronic obstructive } \\
\text { pulmonary disease }\end{array}$ & Yes vs. no & $1.29(0.57-2.92)$ & 0.549 \\
\hline \multicolumn{4}{|l|}{ Coagulopathy } \\
\hline Compensated DICISTH score $\geq 5^{\mathrm{a}}$ & Yes vs. no & $4.58(2.09-10.07)$ & $<0.001$ \\
\hline \multicolumn{4}{|l|}{ Blood coagulation parameters } \\
\hline PT (s) & $\begin{array}{l}\text { Elevated }>3 \text { seconds compared } \\
\text { with the normal limit versus not }\end{array}$ & $2.43(1.15-5.13)$ & 0.021 \\
\hline Fibrinogen $(\mathrm{g} / \mathrm{L})$ & $>4$ vs. $\leq 4$ & $9.50(2.23-40.54)$ & 0.002 \\
\hline D-dimers (ng/mL) & $\begin{array}{l}\text { Elevated vs. normal in } \\
\text { age-specific norm }^{\mathrm{b}}\end{array}$ & $7.65(2.67-21.87)$ & $<0.001$ \\
\hline Protein C (\%) & $<70$ vs. $\geq 70$ & $2.51(1.30-4.83)$ & 0.006 \\
\hline Antithrombin (\%) & $<80$ vs. $\geq 80$ & $2.13(1.18-3.85)$ & 0.012 \\
\hline \multicolumn{4}{|l|}{ Blood cell parameters } \\
\hline Hemoglobin $(\mathrm{g} / \mathrm{dL})$ & $<11$ vs. $\geq 11$ & $2.09(1.18-3.69)$ & 0.011 \\
\hline Hematocrit (\%) & $<40$ vs. $\geq 40$ & $1.58(0.86-2.89)$ & 0.138 \\
\hline Red blood cells $\left(x 10^{9} / \mathrm{L}\right)$ & $<4$ vs. $\geq 4$ & $1.50(0.89-2.52)$ & 0.128 \\
\hline Platelets $\left(\times 10^{9} / \mathrm{L}\right)$ & $<100$ vs. $\geq 100$ & $7.60(1.55-37.35)$ & 0.012 \\
\hline Platelets $\left(\times 10^{9} / \mathrm{L}\right)$ & $<150$ vs. $\geq 150$ & $3.54(1.77-7.10)$ & $<0.001$ \\
\hline White blood cells $\left(\times 10^{9} / \mathrm{L}\right)$ & $>10$ vs. $\leq 10$ & $1.86(1.04-3.33)$ & 0.036 \\
\hline Neutrophils $\left(\times 10^{9} / \mathrm{L}\right)$ & $>7$ vs. $\leq 7$ & $2.43(1.42-4.18)$ & 0.001 \\
\hline Lymphocytes $\left(\times 10^{9} / \mathrm{L}\right)$ & $<1.5$ vs. $\geq 1.5$ & $3.37(1.58-7.21)$ & 0.002 \\
\hline Lymphocytes $\left(\times 10^{9} / \mathrm{L}\right)$ & $<1$ vs. $\geq 1$ & $2.49(1.48-4.20)$ & 0.001 \\
\hline Monocytes $\left(\times 10^{9} / \mathrm{L}\right)$ & $>1$ vs. $\leq 1$ & $0.84(0.35-1.99)$ & 0.687 \\
\hline Eosinophils $\left(\times 10^{9} / \mathrm{L}\right)$ & $>0.07$ vs. $\leq 0.07$ & $0.56(0.30-1.03)$ & 0.060 \\
\hline Basophils $\left(\times 10^{9} / \mathrm{L}\right)$ & $>0.01$ vs. $\leq 0.01$ & $0.69(0.41-1.16)$ & 0.160 \\
\hline
\end{tabular}

Abbreviations: $\mathrm{Cl}$, confidence interval; DIC, disseminated intravascular coagulation; ISTH, International Society on Thrombosis and Haemostasis; PT, prothrombin time.

Note: Values are odds ratio and $95 \%$ confidence intervals.

${ }^{a}$ Assuming COVID-19 is associated with DIC, therefore a +2 term was added for all participants.

${ }^{b}$ Age-adapted threshold for D-dimers: $>500$ for patients under 60 years, $>600$ for age $60-69,>700$ for age $70-79,>800$ for age $80-89$, and $>900$ for age 90-99. 


\section{Hematological Predictors for Disease Worsening}

Increase of fibrinogen $(\mathrm{OR}=9.50,95 \% \mathrm{CI}: 2.23-40.54)$ and D-dimers levels ( $\mathrm{OR}=7.65,95 \% \mathrm{CI}: 2.67-21.87)$, a platelet count lower than $100 \times 10^{9} / \mathrm{L}(\mathrm{OR}=7.60,95 \% \mathrm{CI}: 1.55-37.35)$, and a positive compensated DIC-ISTH score ( $\mathrm{OR}=4.58,95 \% \mathrm{CI}$ : 2.0910.07) were major determinants of disease worsening risk. Deficiency of AT activity (OR=2.13, 95\%CI: 1.18-3.85), PT prolongation $(\mathrm{OR}=2.43,95 \% \mathrm{CI}: 1.15-5.13)$, leukocytosis ( $\mathrm{OR}=1.86,95 \% \mathrm{CI}: 1.04-3.33)$, and lymphopenia with lymphocyte count lower than $1.5 \times 10^{9} / \mathrm{L}(\mathrm{OR}=3.37$, CI: $1.58-7.21)$ were significant risk factors for worsening disease ( - Table 3 ).

\section{COMPASS-COVID-19 Score}

A multivariate logistic regression analysis led to the derivation of a risk assessment model (RAM) for the identification of COVID-19 patients at high risk for worsening disease. The multivariate analysis retained obesity (BMI $\geq 30 ; \mathrm{OR}=6.56$, 95\% CI: 2.98-14.46; $p<0.001)$, male gender ( $\mathrm{OR}=2.59,95 \%$ CI: $1.29-5.21 ; p=0.007)$, compensated DIC-ISTH score $\geq 5$ ( $\mathrm{OR}=2.58,95 \% \mathrm{CI}: 1.07-6.21 ; p=0.034)$, lymphocyte count $<1 \times 10^{9} / \mathrm{L}(\mathrm{OR}=2.21,95 \% \mathrm{CI}: 1.17-4.19 ; p=0.015)$, and $\mathrm{Hb}<11 \mathrm{~g} / \mathrm{dL}(\mathrm{OR}=2.25,95 \% \mathrm{Cl}: 1.13-4.48 ; p=0.021)$ as significant predictors of worsening disease. Multivariate logistic regression analysis led to the following equation:

$\log$ (odds for worsening disease) $=-2.6+1.9^{*}$ (obesity) $+1.0 *$ (male gender $)+0.9 *$ (DIC-ISTH score $\geq 5)+0.8$ (lymphocytes $\left.<1 \times 10^{9} / \mathrm{L}\right)+0.8^{*}(\mathrm{Hb}<11 \mathrm{~g} / \mathrm{dL})$.

The COMPASS-COVID-19 RAM was formulated by calculating an integer numeric value for each predictor according to the value of its multiple regression coefficients ( - Table 4 ). The score ranged between 0 and 54 points with a cut-off at 18 points and stratified COVID-19 patients into high and low risk for worsening disease. The COMPASS-COVID-19 score calcu-
Table 5 Simplified profile of patients with COVID-19 at high or low risk for disease worsening according to the COMPASSCOVID-19 risk assessment model

\begin{tabular}{|c|}
\hline $\begin{array}{l}\text { Patients with COVID-19 at high risk for disease worsening } \\
\text { (COMPASS-COVID-19 score } \geq 18 \text { ) }\end{array}$ \\
\hline Obese (BMI $>30$ ), any sex, any examined comorbidities \\
\hline $\begin{array}{l}\text { Nonobese, male with one or more of: compensated DIC- } \\
\text { ISTH } \geq 5 \text {, lymphopenia, anemia }{ }^{\text {a }}\end{array}$ \\
\hline $\begin{array}{l}\text { Nonobese female with all three of: compensated DIC-ISTH } \\
\geq 5, \text { lymphopenia and anemia }{ }^{\text {a }}\end{array}$ \\
\hline $\begin{array}{l}\text { Patients with COVID-19 at low risk for disease worsening } \\
\text { (COMPASS-COVID-19 score }<18 \text { ) }\end{array}$ \\
\hline $\begin{array}{l}\text { Nonobese male, compensated DIC-ISTH }<5, \text { without } \\
\text { lymphopenia, without anemia }\end{array}$ \\
\hline Nonobese female with none, one, or two of: compensated \\
DIC-ISTH $\geq 5$, lymphopenia, anemia
\end{tabular}

Abbreviations: BMI, body mass index; DIC, disseminated intravascular coagulation; ISTH, International Society on Thrombosis and Haemostasis.

lator is available online at the web site: www.medupdate.eu. - Table 5 shows a simplified profile of patients with COVID-19 at high risk (score $\geq 18$ ) or low risk (score $<18$ ) of disease worsening.

Qualitative Characteristics of the COMPASS-COVID-19 Score The COMPASS-COVID-19 score at the cut-off value for highrisk level ( $\geq 18)$ had $81 \%$ sensitivity, $60 \%$ specificity, $88 \% \mathrm{NPV}$, and $47 \%$ PPV. According to the Hosmer-Lemeshow test, a $p=0.797$ showed that the score was well calibrated. Plotting the expected worsening cases, according to the score, against the observed worsening cases, as well as the expected against

Table 4 COMPASS-COVID-19 score for the evaluation of the risk for worsening disease in COVID-19 patients

\begin{tabular}{|c|c|c|}
\hline \multicolumn{3}{|l|}{ COMPASS-COVID-19 RAM } \\
\hline \multicolumn{2}{|l|}{ Predictors for risk of worsening disease } & Score \\
\hline \multicolumn{2}{|l|}{ Obesity (BMI > 30) } & 19 \\
\hline \multicolumn{2}{|l|}{ Male gender } & 10 \\
\hline \multicolumn{2}{|l|}{ Compensated DIC-ISTH score $\geq 5$} & \multirow[t]{8}{*}{9} \\
\hline Confirmed COVID-19 & 2 & \\
\hline Thrombocytopenia (platelets $<100,000 / \mu \mathrm{L}$ ) & 1 & \\
\hline Prothrombin time prolongation (> control $+3 \mathrm{~s}$ ): & 1 & \\
\hline $\begin{array}{l}\text { D-dimer increase }(>500 \text { for age }<60 \mathrm{y} ;>600 \mathrm{ng} / \mathrm{mL} \text { for age } 60-59 \mathrm{y} \\
>600 \mathrm{ng} / \mathrm{mL} \text { for age } 60-69 \mathrm{y} ;>700 \mathrm{ng} / \mathrm{mL} \text { for age } 70-79 \mathrm{y} \\
>800 \mathrm{ng} / \mathrm{mL} \text { for age } 80-89 \mathrm{y} ;>900 \mathrm{ng} / \mathrm{mL} \text { for age } 90-99 \mathrm{y})\end{array}$ & 1 & \\
\hline Antithrombin decrease ( $<$ lower normal limit established by the laboratory) & 1 & \\
\hline Protein C decrease ( $<$ lower normal limit established by the laboratory) & 1 & \\
\hline Total & $\geq 5$ & \\
\hline \multicolumn{2}{|l|}{ Lymphocytes $<10^{9} / \mathrm{L}$} & 8 \\
\hline \multicolumn{2}{|l|}{ Hemoglobin $<11 \mathrm{~g} / \mathrm{dL}$} & 8 \\
\hline \multicolumn{2}{|l|}{ Total } & $\begin{array}{l}\geq 18 \text { : high risk } \\
<18 \text { : low risk }\end{array}$ \\
\hline
\end{tabular}

Abbreviations: BMI, body mass index; DIC, disseminated intravascular coagulation; ISTH, International Society on Thrombosis and Haemostasis. 


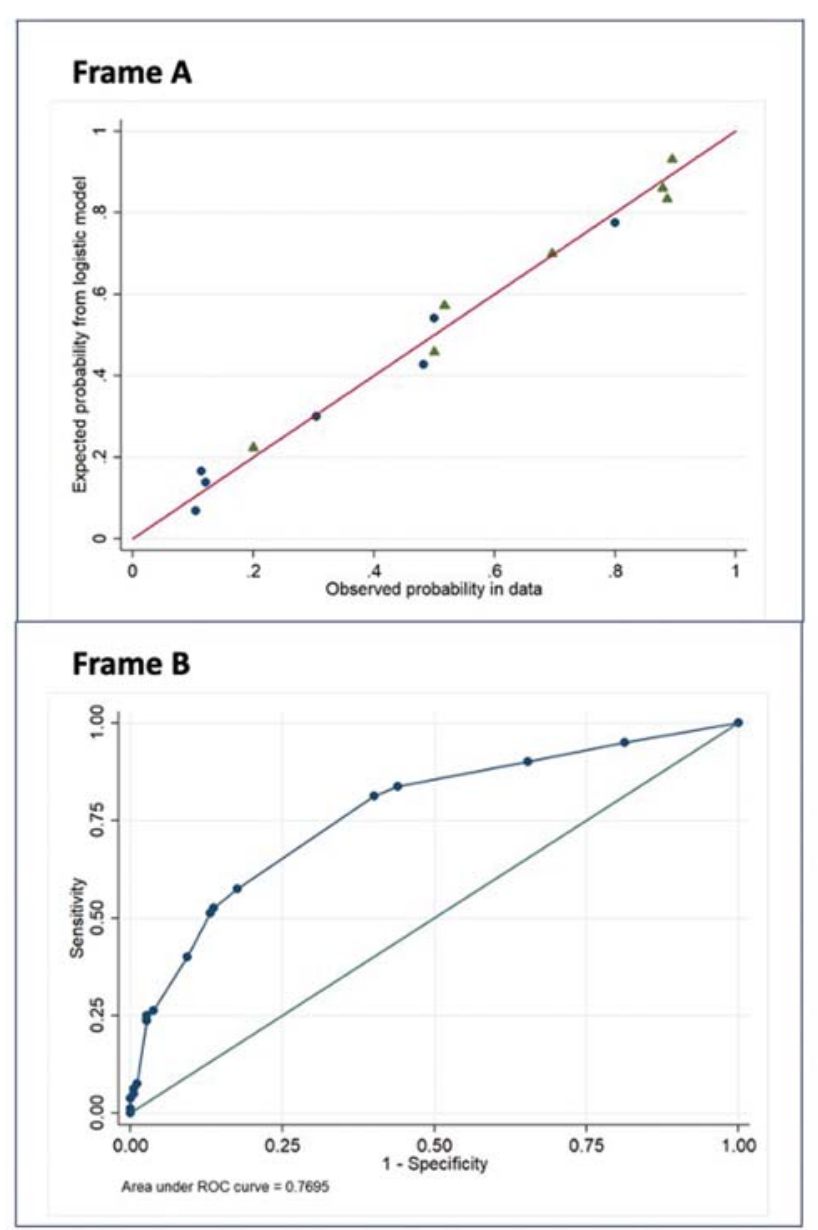

Fig. 1 Qualitative characteristics of the COMPASS-COVID-19 score. Frame A: plot presenting the expected (through the logistic regression equation underlying the score) versus observed probability in worsening cases (circles) and nonworsening cases (triangles). Frame B: the ROC analysis of the model (area under the curve $=0.77$ ).

the observed number of patients whose condition did not worsen, confirmed the good calibration of the score with a Pearson's $r^{2}=0.965$ for worsening cases and $r^{2}=0.992$ for nonworsening cases (-Fig. 1, Frame A). The ROC curve was plotted to evaluate the discrimination ability of the score between the high-risk and low-risk populations for disease deterioration. The AUC was equal to 0.77 , indicating a very good discrimination capacity (-Fig. 1, Frame B). The model with the score minimized the AIC (AIC = 1.033) compared with all other examined logistic regression models, including a univariate model based on the DIC-ISTH score (AIC $=1.191$ ), denoting the substantial improvement through the addition of parameters such as obesity, gender, lymphocyte count, and hemoglobin levels, adopted in the COMPASS-COVID-19 score.

\section{Validation of the COMPASS-COVID-19 Score}

Patients included in the validation cohort were prospectively assessed with the COMPASS-COVID-19 score. The score at the cut-off value of 18 points identified as high risk for disease worsening; $90 \%$ of patients at the W-group and $38 \%$ of the patients at the G-group. The sensitivity and the specificity of the score were 94 and $58 \%$ respectively and the NPV and PPVs were 96 and 45\%, respectively.

\section{Discussion}

Development of prognostic tools and biomarkers for the prediction of COVID-19 trajectory from the time of symptom onset is a difficult task needing urgent response. ${ }^{15,16}$ To anticipate this challenge, we performed this prospective observational cohort study which led to the derivation and validation of the COMPASS-COVID-19 RAM.

We showed that in COVID-19 patients disease worsening is related to the presence of cardiovascular risk factors (i.e., arterial hypertension, diabetes, and obesity) and blood hypercoagulability. The present study showed for the first time that compensated DIC, diagnosed according to the ISTH criteria, $^{23}$ was already present in 8 and $28 \%$ of COVID-19 patients when admitted at the medical conventional ward and the ICU, respectively. Thus, compensated DIC is an independent risk factor for disease worsening. This figure completes the substantial role of blood coagulation activation and DIC in the poor prognosis of COVID-19 patients. ${ }^{27,28}$ Our study underlines that COVID-19 is associated with enhanced blood hypercoagulability documented by the consumption of natural coagulation inhibitors (particularly AT) and the marked increase of D-dimers. This concept is further supported by data from recent postmortem analysis in COVID-19 patients that showed endothelial cell activation and microcirculation abnormalities implicating blood hypercoagulability in the process of disease aggravation. ${ }^{29-31}$ Consequently, our data justify the monitoring of hypercoagulability biomarkers and the need for an early application of antithrombotic treatment in COVID-19 patients. Moreover, close monitoring of AT levels-the most potent heparin cofactor-and its administration in the case of deficiency is mandatory to preserve the treatment efficacy of LMWH. In fact, intravenous administration of AT concentrates could be an effective supportive strategy for the management of DIC in patients with severe COVID-19. ${ }^{14}$

Our study led to the derivation of the COMPASS-COVID-19 score which includes the following easily assessable predictors: presence of obesity (BMI $\geq 30$ ), gender, hemoglobin, lymphocyte count, platelet count, PT, D-dimers, AT, and PC activity. The COMPASS-COVID-19 score accurately identified COVID-19 patients at high risk for disease worsening. The hematological predictors of the score can be easily measured in nonspecialized hematological laboratories. This score is feasible in all health care structures equipped with a routine hematological laboratory. At the cut-off of 18 points, the score has a very good discriminating capacity to stratify patients at high and low risks for disease aggravation, with an AUC value of 0.77 , a sensitivity of $81 \%$, and a specificity of $60 \%$. These qualitative characteristics together with the feasibility of measuring routine hematological parameters designate the COMPASS-COVID-19 score as a useful clinical tool, promptly identifying at least $80 \%$ of patients in the medical ward as being at high risk for disease worsening and as requiring an optimized targeted management. The COMPASS-COVID-19 score 
calculator is available online at the web site www.medupdate. eu. The COMPASS-COVID-19 score for the prediction of disease worsening in hospitalized patients was developed according the TRIPOD reporting guidelines. ${ }^{32}$ Data analysis was performed taking into consideration the major conclusions of the systematic review and critical appraisal of prediction models for diagnosis and prognosis of COVID-19 infection published by Wynants et al. ${ }^{33}$ The prospective design of our study is a strength for the derivation of the new RAM since all patients were tested with the COAG-COVID panel which provided information on hematological alterations together with specific evaluation of biomarkers of hypercoagulability. Moreover, this design allowed the evaluation of the presence of compensated DIC on the first hospitalization day of patients either at the conventional COVID-19 medical ward or at the ICU. The COMPASS-COVID-19 score is not applicable in patients receiving anticoagulant treatment with direct oral anticoagulants or vitamin $\mathrm{K}$ antagonists because these antithrombotic agents introduce prolongation of PT. Moreover, treatment with direct oral anticoagulants (DOACs) induces a variable degree of overestimation of PC activity when a clotting based assay is used. However, the low number of patients with COVID-19 on anticoagulant treatment admitted in our center did not allow the evaluation of the abovementioned conditions on the accuracy of the COMPASSCOVID-19 score. This is an issue that has to be evaluated in the external validation of the score.

Time to event analysis is considered to be the optimal methodology for the elaboration of predictive scores allowing for administrative censoring in a competing risk framework. ${ }^{33}$ However, this strategy was practically unfeasible during the actual phase of the pandemic, which had an extreme pressure on our hospital and on the availability of ICU beds in the city of Paris. For this reason, the cohort design was selected.

External validation is the optimal strategy to control the accuracy of predictive models. However, in the actual phase of the pandemic, this validation strategy is practically impossible to apply. For this reason, we set up an independent validation cohort of patients selected from new patients hospitalized at the COVID-16 center at Tenon University hospital between April 6 and April 21. The assessment of patients enrolled in the validation cohort showed that the COMPASS-COVID-19 score accurately predicted patients at high risk for disease worsening with a very high sensitivity reaching up to $96 \%$. An independent multicenter external validation of the COMPASS-COVID-19 score is ongoing.

Demographics and epidemiological characteristics of the patients enrolled in our study were similar to those described in recently published studies from United States and China. ${ }^{4,34-37}$ Moreover, the low frequency of active smokers found in our cohorts was also reported in recent studies. ${ }^{34,38}$ These similarities further support that patients enrolled in our cohorts are representative of those suffering from COVID-19 in the community and support the generalizability of our findings. Moreover, very limited exclusion criteria were applied, yielding our cohort representative of the population of COVID-19 patients requiring hospitalization.
These characteristics of our study allow implementation of the COMPASS-COVID-19 score across different settings and populations.

We aimed to derive an original, simple, and easy-to-use RAM based on clinical predictors and concrete hematological parameters closely related to mechanisms implicated in COVID-19 pathogenesis. Indeed, available evidence so far has reinforced the importance of blood coagulation, endothelial cell activation, white blood cell alterations, and hypoxia in the deterioration of COVID-19 patients. An enhanced inflammatory reaction with associated-cytokine storm has a central role in COVID-19 patients' worsening. This has also been extensively described in the analysis of biochemical biomarkers of inflammation such as ferritin. Nevertheless, the inflammatory process is reflected upon some of the hematological parameters studied, such as fibrinogen levels, platelets, and white blood cell counts. To the best of our knowledge, two studies have been published to date aiming to elaborate a prediction tool for disease severity in patients hospitalized with COVID-19 and are based on the evaluation of biochemical biomarkers. ${ }^{39,40}$ Both studies included very limited numbers of patients, which hardly allowed (if so) sufficient statistical power to identify score predictors by applying the rule-of-thumb (the so-called EPV 10-1) method for predictive score derivation. In contrast, the COMPASSCOVID-19 score derived from a robust cohort with a sufficient number of patients in the two groups allowing accurate identification of the most pertinent biological and clinical predictors by following the above-mentioned rule.

Despite its original nature, this study bears some limitations. First, in our approach, an unweighted Youden's index was used to establish the optimal cut-off in the score, allocating equal importance to sensitivity and specificity, and yielding a sensitivity of $81 \%$ and a specificity of $60 \%$; alternative, weighted approaches prioritizing for instance sensitivity over specificity could lead to other cut-off values. ${ }^{41}$ Another limitation pertains to the number of patients and the single-center design of the study as well as the short admission time, which were imposed by the urgent character of the SARS-CoV-2 epidemic.

Regarding the external validity and generalizability of findings, it should be underlined that the COMPASSCOVID-19 score is applicable in patients receiving heparin treatment since all patients hospitalized in a conventional medical department or in an ICU routinely receive thromboprophylaxis with body weight-adapted enoxaparin as in our setting. At the actual phase of the score development, the score is not applicable to pregnant women, patients on anticoagulant treatment with VKA or DOAC, and patients with cytopenia due to current anticancer treatment, since these groups of patients were excluded from this study. The applicability of the score on these special groups of patients will be explored in the forthcoming studies.

In conclusion, the present study provides an accurate RAM for early identification of patients with COVID-19 being at high risk of disease worsening that responds to the criteria established by the TRIPOD guidelines. Contextualized application of the COMPASS-COVID-19 score will provide a useful 
clinical decision-making tool for earlier and targeted application of treatments including antithrombotic agents. As stated by Wang et al in a recently published clinical trial on the efficacy and safety of remdesivir, earlier administration of antiviral drugs might be a strategy for successful phase III trials. $^{42}$ The COMPASS-COVID-19 score will be a helpful tool for the identification of patients eligible for phase III trials. The COMPASS-COVID-19 score is based on the presence of pertinent clinical risk factors such as obesity and male gender and also on simple, easy-to-measure hematological and blood coagulation biomarkers, and it can be applied at any level of the health care system. Implementation of the COMPASS-COVID-19 score will allow an easy and rapid identification of a great majority of COVID-19 patients at risk for disease worsening and who may shortly require ICU admission.

\section{What is known about this topic?}

- Development of prognostic tools for the prediction of COVID-19 trajectory from the time of symptom onset is recommended by expert consensus.

\section{What does this paper add?}

- The COMPASS-COVID-19 score derived from a prospective study is composed of easily assessable clinical and hematological predictors.

- The COMPASS-COVID-19 score has high sensitivity (81\%) for the identification of hospitalized patients at high risk of disease worsening.

\section{Data Sharing}

After approval from the legal authorities of the Assistance Publique-Hôpitaux de Paris (APHP), data can be sharedafter contacting the corresponding author (grigorios.gerotziafas@inserm.fr)-with qualifying researchers who submit a proposal with a valuable research question. A contract should be signed.

\section{Authors' Contributions}

G.T.G. as principal investigator has made substantial contributions to the conception and design of the study and analysis and interpretation of the data, wrote the manuscript and has given the final approval of the version to be published, agreed to be accountable for all aspects of the work in ensuring that questions related to the accuracy or integrity of any part of the work are appropriately investigated and resolved. T.N.S. has made substantial contribution by doing the statistical plan and the statistical analysis. G.V. has made substantial contribution in the enrolment of the patients and participated in the design of the study. L.L. has made substantial contribution in the enrolment of the patients. C.P. has made substantial contribution in data acquisition, data interpretation, and critical revision of the manuscript. A.E. participated in the enrollment of the patients and data acquisition. M. $\mathrm{T}$. participated in the enrollment of the patients and data acquisition. P.V. participated in the critical revision and editing of the manuscript. L.P. participated in the critical revision and editing of the manuscript. T.P. participated in the statistical analysis. E.T. critically revised the manuscript. M-.A.D. gave valuable suggestions for data analysis and critically revised the manuscript. A.P., J.C., and G.P. have made substantial contribution in the enrollment of the patients and critically revised the manuscript. M.F. made substantial contribution to the design of the study, the interpretation of the data, and critically revised the manuscript. I.E. critically revised the manuscript.

\section{Conflict of Interest}

None declared.

\section{References}

1 Wu F, Zhao S, Yu B, et al. A new coronavirus associated with human respiratory disease in China. Nature 2020;579(7798):265-269

2 Lu R, Zhao X, Li J, et al. Genomic characterisation and epidemiology of 2019 novel coronavirus: implications for virus origins and receptor binding. Lancet 2020;395(10224):565-574

3 Wan Y, Shang J, Graham R, Baric RS, Li F. Receptor recognition by the novel coronavirus from Wuhan: an analysis based on decadelong structural studies of SARS coronavirus. J Virol 2020;94(07): e00127-20

4 Huang C, Wang Y, Li X, et al. Clinical features of patients infected with 2019 novel coronavirus in Wuhan, China. Lancet 2020;395 (10223):497-506

5 Chen N,Zhou M, Dong X, et al. Epidemiological and clinical characteristics of 99 cases of 2019 novel coronavirus pneumonia in Wuhan, China: a descriptive study. Lancet 2020;395(10223):507-513

6 Driggin E, Madhavan MV, Bikdeli B, et al. Cardiovascular considerations for patients, health care workers, and health systems during the COVID-19 pandemic. J Am Coll Cardiol 2020;75(18):2352-2371

7 Bangash MN, Patel J, Parekh D. COVID-19 and the liver: little cause for concern. Lancet Gastroenterol Hepatol 2020;5(06):529-530

8 Mehta P, McAuley DF, Brown M, Sanchez E, Tattersall RS, Manson JJHLH Across Speciality Collaboration, UK. COVID-19: consider cytokine storm syndromes and immunosuppression. Lancet 2020;395(10229):1033-1034

9 Bikdeli B, Madhavan MV, Jimenez D, et al. COVID-19 and thrombotic or thromboembolic disease: implications for prevention, antithrombotic therapy, and follow-up: JACC state-of-the-art review. J Am Coll Cardiol 2020;75(23):2950-2973

10 Thachil J, Tang N, Gando S, et al. ISTH interim guidance on recognition and management of coagulopathy in COVID- 19 . J Thromb Haemost 2020;18(05):1023-1026

11 Kollias A, Kyriakoulis KG, Dimakakos E, Poulakou G, Stergiou GS, Syrigos K. Thromboembolic risk and anticoagulant therapy in COVID-19 patients: emerging evidence and call for action. Br J Haematol 2020;189(05):846-847

12 Onder G, Rezza G, Brusaferro S. Case-fatality rate and characteristics of patients dying in relation to COVID-19 in Italy. JAMA 2020;323(18):1775-1776

13 Poston JT, Patel BK, Davis AM. Management of critically ill adults with COVID-19. JAMA 2020;323(18):1839-1841

14 Terpos E, Ntanasis-Stathopoulos I, Elalamy I, et al. Hematological findings and complications of COVID-19. Am J Hematol 2020;95 (07):834-847

15 Li X, Xu S, Yu M, et al. Risk factors for severity and mortality in adult COVID-19 inpatients in Wuhan. J Allergy Clin Immunol 2020;146(01):110-118 
16 Phua J, Weng L, Ling L, et al. Intensive care management of coronavirus disease 2019 (COVID-19): challenges and recommendations. Lancet Respir Med 2020;8(05):506-517

17 Cao B, Wang Y, Wen D, et al. A trial of lopinavir-ritonavir in adults hospitalized with severe Covid-19. N Engl J Med 2020;382(19): 1787-1799

18 Shen C, Wang Z, Zhao F, et al. Treatment of 5 critically ill patients with COVID-19 with convalescent plasma. JAMA 2020;323(16): 1582-1589

19 Alexander PE, Debono VB, Mammen MJ. COVID-19 coronavirus research has overall low methodological quality thus far: case in point for chloroquine/hydroxychloroquine.J Clin Epidemiol 2020; 123:120-126

20 Baden LR, Rubin EJ. Covid-19-the search for effective therapy. N Engl J Med 2020;382(19):1851-1852

21 Grein J, Ohmagari N, Shin D, et al. Compassionate use of remdesivir for patients with severe Covid-19. N Engl J Med 2020;382 (24):2327-2336

22 Rubin GD, Ryerson CJ, Haramati LB, et al. The role of chest imaging in patient management during the COVID-19 Pandemic: a multinational consensus statement from the Fleischner Society. Radiology 2020;296(01):172-180

23 Taylor FB, Toh CH, Hoots WK, Wada H, Levi MScientific subcommittee on disseminated intravascular coagulation (DIC) of the International Society on Thrombosis and Haemostasis (ISTH). Towards definition, clinical and laboratory criteria, and a scoring system for disseminated intravascular coagulation. Thromb Haemost 2001;86(05):1327-1330

24 Hendriksen JM, Geersing GJ, Moons KG, de Groot JA. Diagnostic and prognostic prediction models. J Thromb Haemost 2013;11 (Suppl 1):129-141

25 Harrell FE Jr, Lee KL, Mark DB. Multivariable prognostic models: issues in developing models, evaluating assumptions and adequacy, and measuring and reducing errors. Stat Med 1996;15(04): 361-387

26 Peduzzi P, Concato J, Kemper E, Holford TR, Feinstein AR. A simulation study of the number of events per variable in logistic regression analysis. J Clin Epidemiol 1996;49(12):1373-1379

27 Tang N, Li D, Wang X, Sun Z. Abnormal coagulation parameters are associated with poor prognosis in patients with novel coronavirus pneumonia. J Thromb Haemost 2020;18(04):844-847

$28 \mathrm{Wu} \mathrm{C}$, Chen X, Cai Y, et al. Risk factors associated with acute respiratory distress syndrome and death in patients with coronavirus disease 2019 pneumonia in Wuhan, China. JAMA Intern Med 2020;180(07):1-11

29 Varga Z, Flammer AJ, Steiger P, et al. Endothelial cell infection and endotheliitis in COVID-19. Lancet 2020;395(10234):1417-1418
30 Du Y, Tu L, Zhu P, et al. Clinical features of 85 fatal cases of COVID19 from Wuhan. A retrospective observational study. Am J Respir Crit Care Med 2020;201(11):1372-1379

31 Cattaneo M, Bertinato EM, Birocchi S, et al. Pulmonary embolism or pulmonary thrombosis in COVID-19? Is the recommendation to use high-dose heparin for thromboprophylaxis justified? Thromb Haemost 2020;120(08):1230-1232

32 Moons KGM, Altman DG, Reitsma JB, et al. Transparent Reporting of a multivariable prediction model for Individual Prognosis or Diagnosis (TRIPOD): explanation and elaboration. Ann Intern Med 2015;162(01):W1-73

33 Wynants L, Van Calster B, Collins GS, et al. Prediction models for diagnosis and prognosis of covid-19 infection: systematic review and critical appraisal. BMJ 2020;369:m1328

34 Richardson S, Hirsch JS, Narasimhan M, et al; and the Northwell COVID-19 Research Consortium. Presenting characteristics, comorbidities, and outcomes among 5700 patients hospitalized with COVID-19 in the New York City Area. JAMA 2020;323(20): 2052-2059

35 Garg S, Kim L, Whitaker M, et al. Hospitalization rates and characteristics of patients hospitalized with laboratory-confirmed coronavirus disease 2019 - COVID-NET, 14 states, March 1-30, 2020. MMWR Morb Mortal Wkly Rep 2020;69 (15):458-464

36 Zheng Z, Peng F, Xu B, et al. Risk factors of critical \& mortal COVID19 cases: a systematic literature review and meta-analysis. J Infect 2020;81(12):e16-e25

37 Mehra MR, Desai SS, Kuy S, et al. Cardiovascular disease, drug therapy, and mortality in Covid-19. N Engl J Med 2020; 382:e102

38 Miyara M, Tubach F, Pourcher V, et al. Low incidence of daily active tobacco smoking in patients with symptomatic COVID-19. Qeios 2020. Doi: $10.32388 / W P P 19 W .3$

39 Bai X, Fang C, Zhou Y, et al. Predicting COVID-19 malignant progression with AI techniques. medRxiv 2020. Doi: 10.1101/ 2020.03.20.20037325

40 Gong J, Ou J, Qiu X, et al. A tool for early prediction of severe coronavirus disease 2019 (COVID-19): a multicenter study using the risk nomogram in Wuhan and Guangdong, China. Clin Infect Dis 2020;71(15):833-840

41 Li DL, Shen F, Yin Y, Peng JX, Chen PY. Weighted Youden index and its two-independent-sample comparison based on weighted sensitivity and specificity. Chin Med J (Engl) 2013;126(06): $1150-1154$

42 Wang Y, Zhang D, Du G, et al. Remdesivir in adults with severe COVID-19: a randomised, double-blind, placebo-controlled, multicentre trial. Lancet 2020;395(10236):P1569-P1578 